\title{
Diagnostic skill and patient centred care
}

\author{
Fiona Godlee editor in chief, The BMJ
}

\begin{abstract}
"Overdiagnosis of the well and undertreatment of the sick are the conjoint twins of modern medicine." So writes Iona Heath in an essay this week (doi:10.1136/bmj.g6123). She articulates the fears that drive medical excess: our existential fear of dying, and the ever present fear that a serious diagnosis will be missed. While striving for accurate diagnoses and appropriate treatment, Heath says that clinicians and patients need to acknowledge the limits of medicine and embrace the uncertainty that lies beyond. "Only because we do not understand everything and because we cannot control the future is it possible to live," she says.

Faced with uncertainty and the fear of uncertainty, clinicians may find themselves ordering batteries of tests, perhaps especially younger clinicians and those working in more litigious environments. The avoidable cost and harm that can follow are only now being properly understood. But the best medicine still relies on a careful history and examination. This week's journal reviews three conditions in which good clinical diagnosis is the key to appropriate treatment.
\end{abstract}

As shown in our clinical review by Scott Middleton and Raymond Anakwe (doi:10.1136/bmj.g6437), diagnosing carpal tunnel syndrome allows you to practise the art of medicine while reviving an affection for medical history: who were Tinel, Phalen, and Durkan of the eponymous signs and tests?

Nasal septal haematoma may sound trivial, but if undetected it can lead to irreversible septal necrosis and severe cosmetic distortion of the nose. A history of nasal trauma, no matter how trivial, should prompt inspection of the nasal septum, say Leigh Sanyaolu and colleagues (doi:10.1136/bmj.g6075), especially if followed by symptoms of nasal obstruction, pain, rhinorrhoea, or fever.

Finally, and much less common, is spontaneous intracranial hypotension. Shona Scott and Richard Davenport report that the cardinal symptom is a debilitating postural or low pressure headache, relieved by lying down and worsened by being upright (doi:10.1136/bmj.g6219). Confirmation is by magnetic resonance imaging of the brain. Treatment is conservative or, if that fails, at best experimental.

Scott and Davenport's article is itself experimental. It is our first to include a box describing how patients were involved in the creation of the article. You'll see more of these boxes in future as part of our commitment to co-create content. Our aim is not only to be more relevant and patient centred but to give credibility to the journal's calls for co-creation of healthcare. There is much work to do. A new report from the King's Fund finds that UK healthcare is falling short on the leadership and culture change needed to truly empower patients (doi:10.1136/ bmj.g6613). There has been some progress, it says, mainly in how patients are involved in research, and this is an area where we hope The BMJ can also help. As well as patients peer reviewing research papers, we now ask authors to say whether they involved patients, service users, carers, or lay people in the design and implementation of their study. Look out for summaries of what authors say in forthcoming research articles.

Cite this as: BMJ 2014;349:96665

๑ BMJ Publishing Group Ltd 2014 